\title{
Molecular gas across cosmic time
}

\author{
Georgios E. Magdis ${ }^{1,2,3}$ (i) \\ ${ }^{1}$ Cosmic Dawn Center (DAWN) \\ ${ }^{2}$ Niels Bohr Institute, University of Copenhagen, Lyngbyvej 2, 2100 Copenhagen, Denmark \\ ${ }^{3}$ Institute for Astronomy, Astrophysics, Space Applications and Remote Sensing, National \\ Observatory of Athens, 15236, Athens, Greece \\ email: georgios.magdis@nbi.ku.dk
}

\begin{abstract}
We have entered an era where the gas mass estimates of distant galaxies do not rely on a single tracer but rather on an inventory of different and independent methods, much like the case for the determination of the star formation rate (SFR) of the galaxies. This is crucial as the traditional $M_{\text {gas }}$ tracers, i.e. low-J CO transition lines and dust continuum emission are becoming highly uncertain as we move to higher redshifts due to metallicity and CMB effects. Here, we present a homogeneous and statistically significant investigation of the use of atomic carbon as an alternative $M_{\text {gas }}$ tracer (Valentino et al. 2018) and provide evidence of optically thick far-IR emission in high $-z$ starbursts that point towards higher dust temperatures and lower dust and gas mass estimates than previously inferred (Cortzen et al. 2019, submitted). Finally, we present direct observations of the effect of the CMB on the far-IR SEDs of high-z SBs, manifested by unphyscally large $(\beta=2.5-3.5)$ apparent spectral indexes in R-J tail (Jin et al. 2019, submitted).
\end{abstract}

Keywords. galaxy evolution, ISM, starbursts

\section{Introduction}

A critical property that dictates galaxy evolution is the amount and the physical conditions of the cold, star forming gas primarily in the form of $\mathrm{H}_{2}$, that is bound within the galaxies. However, since under typical conditions molecular hydrogen cannot be directly observed, in order to measure the amount of gas $\left(M_{\text {gas }}\right)$ in the galaxies, and much like the case of star formation rate (SFR) indicators, we have been are building an inventory of indirect gas tracers and techniques. A collection of such tracers are shown in Figure 1 and summarised below:

- the low-J CO transition lines (e.g. Solomon \& Vanden Bout 2005, Carilli \& Walter 2013)

- the dust continuum emission, either through the metallicity depended dust to gas mass ratio technique (e.g. Magdis et al. 2012) or though the monochromatic flux density in the R-J tail (e.g. Scoville et al. 2017)

- the neutral atomic carbon ([CI]) lines (e.g. Valentino et al. 2018)

- the single ionised atomic carbon line ([CII]) (e.g. Zanella et al. 2018)

- the polycyclic aromatic hydrocarbon (PAHs) emission in the mid-IR (e.g. Cortzen et al. 2019)

- the HCN emission line, sensitive to the dense gas reservoir (e.g. Gao \& Solomon 2004) and

- dynamical analysis (e.g. Tacconi et al. 2008, Daddi et al. 2010)

Applying primarily the first two techniques ( $\mathrm{CO}$ and dust) in large samples of galaxy populations has enable the discovery of various important scaling relations between the 


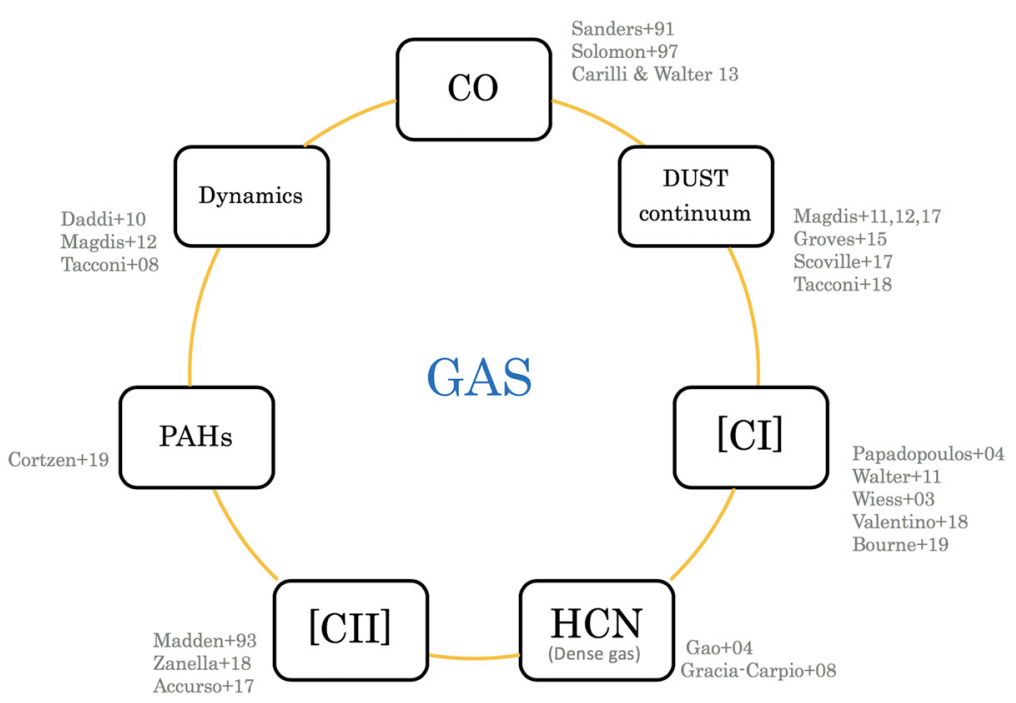

Figure 1. A collection of indirect gas mass tracers that have been employed so far, fo both local and high $-z$ galaxies. The provided references are by far incomplete, but they are indicative of the available literature for each method.

amount of available gas, the stellar mass and the star formation rate of he galaxies that have sed light into the characterisation of the star formation mode, the gas depletion time scales and the star formation efficiency of the galaxies out to $z \sim 3$ and beyond (e.g. Magdis et al. 2012, Sargent et al. 2014, Scoville et al. 2017, Tacconi et al. 2018) Furthermore, these studies have traced the evolution of the gas fraction, and more recently the evolution of the molecular gas density, coupling the well established and more mature studies that have unveiled the evolution of the specific star formation rate and the evolution of the star formation rate density of the Universe. However, while the various empirical and observationally motivated models of the gas content of the galaxies tend to agree up to $\mathrm{z} \sim 3$, the available samples decrease and the uncertainties on how to convert the observable into gas mass estimates increase substantially as we move to higher redshifts, leaving our knowledge of the gas content of the early galaxies poorly constrained.

\section{Caveats and the way forward}

A main caveat of the $\mathrm{CO}$ and dust method to trace gas masses is their dependence on the gas phase metallicity (though the adopted $\alpha_{\mathrm{CO}}$ and $\delta_{\mathrm{G} / \mathrm{D}}$ ) that varies as a function of environment, of redshift but also as a function of star formation mode. Indeed, critical open question that still remains open and has been the topic of hot debate is the variation of the metallicity (and therefore of the appropriate $\alpha_{\mathrm{CO}}$ and $\delta_{\mathrm{G} / \mathrm{D}}$ conversion factors) between the main sequence (MS) and starbursts (SB) galaxies, a that could lead into a bimodal or alternatively a continuous star formation mode in the galaxies (e.g. Daddi et al. 2010, Sargent et al. 2014, Scoville et al. 2017). Another caveat arises from the increase of the temperature and of the background emission of the CMB that makes the detection of the low- $J$ CO transitions and of the and dust continuum emission in the R-J tail against the CMB background extremely challenging at high redshifts (e.g. da Cunha et al. 2013). Finally, until now the dust method has been applied under the implicit assumption that the far-IR emission is optically thin. However, previous studies local ULIRGs and more recent ALMA observations of high-z star-bursting galaxies (Hodge et al. 2016, Simpson et al. 2017) provide evidence that the emission of the galaxies 


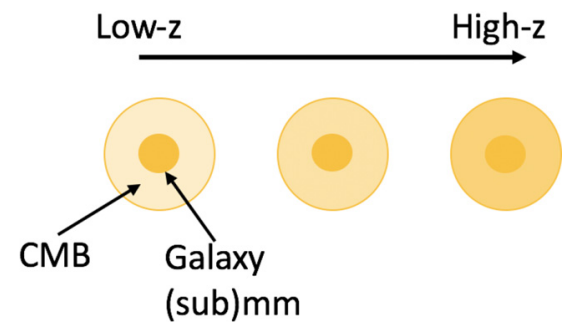

Figure 2. A sketch depicting the effect of the $\mathrm{CMB}$ on the measured dust continuum emission as we move to higher redshifts. The CMB background emission becomes progressively more prominent, washing out the contrast between the galaxy emission and the background.

remains optically thick out to $\lambda_{\text {rest }} 200 \mu m$, with important consequences on the derived dust temperature and therefore the dust (and gas) mass of the galaxies.

An alternative method that has been put forward as a promising gas tracer is the [CI] line (e.g. Papadopoulos et al. 2004) that come with several advantages with respect to the $\mathrm{CO}$ and dust method: 1 ) it is optically thin, 2) cosmic rays tend to dissociate CO to $\mathrm{C}$ leaving $\mathrm{CO}$ dark clouds of gas, 3 ) it is less affected by metallicity and 4) it has a low excitation potential, typical of GMC. However before using [CI] as gas tracer for high-z galaxies we first need to consistently and homogeneously calibrate it among different environments at lower redshifts. In the same direction, it is also important to identify and quantify the effect of the CMB on the dust-continuum emission and also disentangle between optically thin and optically thick far-IR emission and thus yield in more robust dust and gas mass estimates.

\section{ALMA [CI] surveys, CMB effect and dust opacity in the early galaxies}

To assess the potential of $[\mathrm{CI}]$ as a gas mass tracer and to explore how to convert the [CI] line luminosities into $M_{\text {gas }}$ we have been carrying out ALMA Band 6 and Band 7 surveys targeting CI[1-0] emission from a sample of $\sim 70 \mathrm{MS}$ and SBs at $z=0.5-1.5$, that also benefit from robust far-IR properties $\left(T_{\mathrm{d}} L_{\mathrm{IR}} M_{\mathrm{dust}}\right)$ obtained from their well sampled mid to far-IR SEDs from Spitzerand Herschel. Preliminary results published by Valentino et al. 2018, indicate that for fixed $L_{\mathrm{IR}}$, MS galaxies have on average higher CI luminosities with respect to local and high $-z$ SBs something that closely resembles the $L_{\mathrm{CO}}^{\prime}-L_{\mathrm{IR}}$ relation were again MS galaxies here in green exhibit on average higher $L_{\mathrm{CO}}^{\prime} / L_{\mathrm{IR}}$ ratios, indicative of higher $M_{\text {gas }} / \mathrm{SFR}$ therefore of longer gas depletion time scales and lower star formation efficiencies. Most importantly, when the [CI] lines are combined with the traditional $\mathrm{CO}$ and dust-based $M_{\text {gas }}$ estimates, Valentino et al. 2018 reports a $[\mathrm{CI}] /\left[\mathrm{H}_{2}\right]$ abundance ratio of $\sim 1.6-1.9 \times 10^{-5}$ that, for the given set of assumptions is $3-8$ times larger than that of SBs galaxies at any redshift (Figure 3 left).

Another diagnostic power of the neutral atomic carbon is that thanks to its simple 3 level structure the CI[2-1]/CI[1-0] line ratio can be used to infer the excitation temperature of the gas ( $T_{\text {exc }}$, e.g., Weißet al. 2003). Since under LTE conditions $T_{\text {exc }} \approx T_{\text {kin }} \approx$ $T_{\mathrm{d}}$, the line ratio can be used to shed light into the "true" $T_{\mathrm{d}}$ of high $-z$ starbursts that might appear to be cold due to the fact the their far-IR emission remains optically thick out to $\lambda_{\text {rest }} \sim 200-300 \mu \mathrm{m}$ while in fact their ISM is characterised by much a higher $T_{\mathrm{d}}$. To test this, Cortzen et al. 2019 (submitted), used NOEMA observation to measure the $\mathrm{CI}[2-1] / \mathrm{CI}[1-0]$ line ratio of GN20, a template star-bursting galaxy at $z=4.05$ (e.g. Pope et al. 2006, Magdis et al. 2011, Tan et al. 2014) The [CI] data yield a $T_{\mathrm{exc}} \sim 47 \pm 8$ which is much higher than the $T_{\mathrm{d}}$ of $33 \pm 2 \mathrm{~K}$ derived under the assumption of an optically thin 

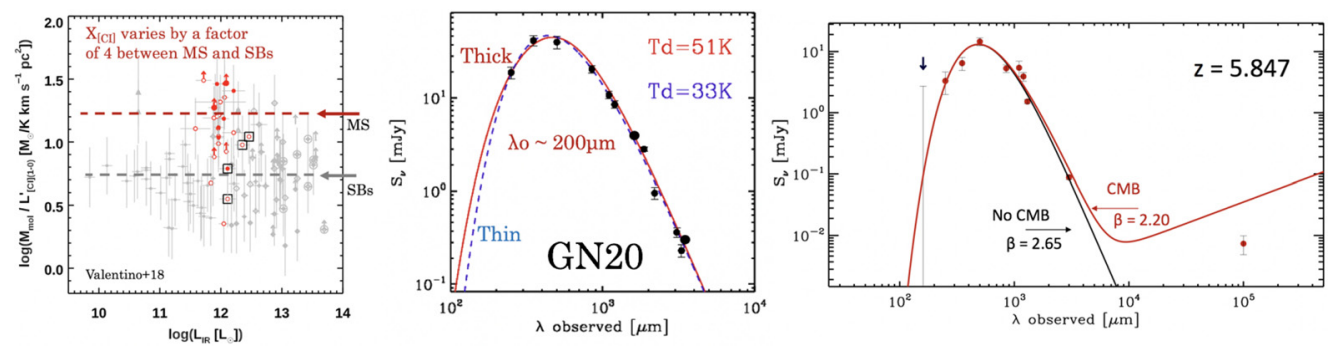

Figure 3. Left) $M_{\text {gas }} /$ lci vs $L_{\mathrm{IR}}$ for local and high-z SBs (grey) as well as z $1.0 \mathrm{MS}$ galaxies from Valentino et al. 2018. Middle) Optically thin and optically thick fit to the far-IR continuum data of GN20. Right) The SED of a SB galaxy from Jin et al. 2019 with the best fit MBB models with and without the effects of the CMB. The black line corresponds to the apparent SED of the source while the red line to the intrinsic SED when corrected for the CMB effects.

modified black body. Instead, they show that when a general opacity model is adopted, the derived $T_{\mathrm{d}}(\sim 52 \pm 4 \mathrm{~K})$ is much closer the one indicated by the [CI] data, suggesting that GN20 becomes optically thin at $\lambda_{\text {rest }} \sim 200 \mu \mathrm{m}$ (Figure 3 middle). This pilot study serves as first evidence that actual $T_{\mathrm{d}}$ high $-z \mathrm{SB}$ galaxies is much warmer than previously thought, providing an elegant solution to the puzzling observation of SBs becoming colder than MS galaxies at $z>2$ and mitigates their unphysically high $M_{\mathrm{dust}} / M_{*}$ ratios reported in the literature (e.g. Bethermin et al. 2015), since higher $T_{\mathrm{d}}$ correspond to lower $M_{\text {dust }}$ (and consequently lower $M_{\text {gas }}$ ) estimates.

These findings are very timely with respect to the recent discovery of a population of very cold high $-z(z \sim 4-6)$ SBs by Jin et al. 2019 (submitted). Using ALMA spectral scanning they confirmed the redshifts of high $-z$, dusty star-bursting candidates in the COSMOS field, which turned out to have (under the opticaly thin assumption) considerably lower dust temperatures with respect to MS galaxies at the same redshifts. An optically thick far-IR emission that would yield higher $T_{\mathrm{d}}$ is a very appealing solution to this puzzling observation, that is also in line with the small/compact sizes of the dust emitting region in these galaxies. Furthermore, the most notable characteristic of these sources is that we find for the first time direct observational evidence of the effect of the $\mathrm{CMB}$ on the R-J continuum emission of high $-z$ galaxies. This is revealed through the uphysically steep spectral indexes $(\beta=2.5-3.5)$ that are recover when CMB effects are neglected. On the other hand, when the $\mathrm{CMB}$ effects are taken into account (following the recipe described in da Cunha et al. 2013), we recover reasonable $\beta$ values in the range of $1.8-2.2$. The SED along with the fits with and without the inclusion of the CMB effects for one for the sources from Jin et al. 2019 is shown in Figure 3 (right).

\section{Summary}

While the $\mathrm{CO}$ and dust methods to trace $M_{\text {gas }}$ has provided a robust and coherent picture out to $z \sim 3$, pushing molecular gas studies at higher redhsifts require alternative techniques mainly due to the dependance of the two methods on metallicity dependance and the CMB effects that hamper the detectability of both dust emission in the R-J regime as well as of the low- $J$ CO lines at high redshifts. Our ALMA surveys, combined with literature data, suggest that the [CI] line that has been put forward as an alternative gas tracer also suffers from similar systematics, with a varying $[\mathrm{CI}] /\left[\mathrm{H}_{2}\right]$ abundance ratio among different galaxy types. On top of the we provide evidence that the far-IR emission of high $-z$ starbursts is optically thick with considerably higher dust temperatures and thus lower $M_{\text {dust }}$ and $M_{\text {gas }}$ estimates than previously inferred. Similarly, we find first direct observations of the impact of the CMB in the dust continuum emission of a population of "apparently" cold $z>3$ SBs thanks to the abnormally high $\beta$ values 
that we recover when fitting the ALMA continuum data in the R-J tail without taking into account the effects of the CMB. When these effects are taken into consideration the derived $\beta$ values are more consistent with the well established $\beta=1.5-2.0$ range.

The presented results are based on the works of Valentino et al. 2018, Jin et al. 2019 (submitted) and Cortzen et al. 2019 (submitted) with invaluable contributions from E. Daddi and D. Liu.

\section{References}

Accurso, G., Saintonge, A., Catinella, B., et al. 2017, MNRAS, 470, 4750

Bethermin, M., Daddi, E., Magdis, G., et al. 2015, A $\mathscr{E} A$, 573, A113

Bourne, N., Dunlop, J. S., Simpson, J. M. et al., 2019, MNRAS, 482, 3135

Carilli, C. L., \& Walter, F. 2013, Annual Review of A\&A, 51, 1

Cortzen, I., Garrett, J., Magdis, G., et al. 2019, MNRAS, 482, 1618

da Cunha, E., Groves, B., Walter, F., et al. 2013, ApJ, 766, 13

Daddi, E., Bournaud, F., Walter, F., et al. 2010, ApJ, 713, 686

Daddi, E., Elbaz, D., Walter, F., et al. 2010, ApJ, 714, L118

Gao, Y. \& Solomon, P. M., 2004, ApJ, 606, 271

Gracia-Carpio, J., Garcia-Burillo \& Santiago, P., et al. 2008, A\&A A, 479, 703

Groves, B., A., Schinnerer, E., Leroy, A., et al. 2015, ApJ, 799, 96

Hodge, J. A., Swinbank, A. M., Simpson, J. M., et al. 2016, ApJ, 833, 103

Madden, S. C., Geis, N., Genzel, R., et al. 1993, ApJ, 407, 579

Jin, et al. 2019, ApJ, 887, 15

Magdis, G., Daddi, E., Elbaz, D., et al. 2011, ApJ, 740, 15

Magdis, G. E., Daddi, E., Bethermin., et al. 2012, ApJ, 760, 6

Magdis, G. E., Rigopoulou, D., Daddi, E., et al. 2017, A\&ऽA, 603, A93

Papadopoulos, P. P., Thi, W. F., \& Viti, S. 2004, MNRAS, 351, 147

Pope, A., Scott, D., Dickinson, M., et al. 2006, MNRAS, 370, 1185

Sanders, D. B., Scoville, N. Z., \& Soifer, B. T., 1991, ApJ, 370, 158

Sargent, M., Daddi, E., Bethermin, M., et al. 2014, ApJ, 793, 19

Scoville, N., Lee, N., Vanden Bout, P., et al. 2017, ApJ, 837, 150

Simpson, J. M., Smail, I., Swinbank, A. M., et al. 2017, ApJ, 839, 58

Solomon, P. M., Downes, D., Radford, S. J. E., et al. 1997, 478, 144

Solomon, P. M., \& Vanden Bout, P. A. 2005, ARAA, 43, 677

Tacconi, L. J., Genzel, R., Smail, I., et al. 2008, ApJ, 680, 246

Tacconi, L. J., Genzel, R., Saintonge, A., et al. 2018, ApJ, 853, 179

Tan, Q., Daddi, E., Magdis, G., et al. 2014, A\&A, 569, A98

Valentino, F., Magdis, G. E., Daddi, E, et al. 2018, ApJ, 869, 27

Walter, F., Weiß, A., Downes, D., et al. 2011, ApJ, 730, 18

Weiß, A., Henkel, C., Downes, D., \& Walter, F. 2003, A\&A, 409, L41

Zanella, A., Daddi, E., Magdis, G., et al. 2018, MNRAS, 481, 1976 